\title{
Serum metabolomic analysis of feline mammary carcinomas based on LC-MS and MRM techniques
}

\author{
Jia-san Zheng*, Ren-yue Wei*, Zheng Wang, \\ Jun Song, Yan-song Ge, Rui $\mathrm{Wu}^{凶}$ \\ College of Animal Science and Veterinary Medicine, \\ Heilongjiang Bayi Agricultural University, Daqing, 163319, China \\ 595095793@qq.com
}

Received: April 16, $2020 \quad$ Accepted: October 7, 2020

\begin{abstract}
Introduction: To date, there have been no panoramic studies of the serum metabolome in feline mammary carcinoma. As the first such study, metabolomics techniques were used to analyse the serum of cats with these tumours. Three important metabolic pathways of screened differential metabolites closely related to feline mammary carcinomas were analysed to lay a theoretical basis for further study of the pathogenesis of these carcinomas. Material and Methods: Blood in a 5-8 mL volume was sampled from twelve cats of the same breed and similar age (close to nine years on average). Six were feline mammary carcinoma patients and six were healthy. L glutamate, L alanine, succinate, adenine, hypoxanthine, and inosine were screened as were alanine, aspartate, and glutamate metabolism, the tricarboxylid acid (TCA) cycle, and purine metabolism. Data were acquired with LC-MS non-target metabolomics, multiple reaction monitoring target metabolomics, and multivariate statistical and bioinformatic analysis. Results: Expression of five of the metabolites was upregulated and only inosine expression was downregulated. Up- and downregulation of metabolites related to glycometabolism, potentiation of the TCA cycle, greater content of lipid mobilisation metabolites, and abnormality of amino acid metabolism were closely related to the occurrence of the carcinomas. Conclusion: These findings provide a new direction for further study of the mechanisms associated with cat mammary neoplasms.
\end{abstract}

Keywords: cats, feline mammary carcinomas, LC-MS, metabolomics, multiple reaction monitoring (MRM).

\section{Introduction}

Feline mammary carcinoma is the third most common cancer that affects female cats (13). Its incidence is closely related to age, breed, hormone levels, and sterilisation (22). The disease is associated with a high metastasis rate and poor prognosis (3). Common metastatic areas include the lymph nodes, lungs, liver, and pleura. Since feline mammary carcinoma exhibits the same histological, molecular, and clinical characteristics as human breast cancer, it is considered to be an appropriate animal model for the study of it (17).

Currently, most research on feline mammary carcinoma has focused on the role that one substance plays in carcinoma development $(2,4,6,7,12)$. In addition, there are few studies on the metabolic changes associated with the carcinomas. To date, there have been no panoramic studies of the serum metabolome in this neoplasm. Metabolites are the downstream products regulated by the living system, which can directly and comprehensively reflect the metabolic state of the organism. Serum samples can be readily obtained in veterinary clinics and are convenient for use as test samples. This study used LC-MS non-target metabolomics and multiple reaction monitoring (MRM) target metabolomics to detect metabolites in the serum of cats with mammary carcinomas. The metabolic pathways of the screened differential metabolites were analysed with the aim of providing a theoretical basis for further study of the pathogenesis of feline mammary carcinomas.

\section{Material and Methods}

Selection and grouping of experimental animals. The test samples were derived from feline mammary 
carcinoma cases treated at the animal hospital of Heilongjiang Bayi Agricultural University. Six Chinese pastoral cats aged 8.83 years on average with cases of mammary carcinomas confirmed by histopathology comprised the experimental group (group T). None of the cats had been treated with chemotherapy or radiotherapy. Another six healthy cats aged 8.67 years on average of the same breed and age served as the control group (group C). Specific information about the experimental and control cats is shown in Table 1.

Sample collection. Before tumour resection, 5-8 $\mathrm{mL}$ of blood was collected from the jugular vein of the experimental group cats and placed in a centrifugal tube without anticoagulant. An equal volume of blood was sampled from the same site of the control group cats. A set of six samples from each of the two groups was placed in a refrigerator at $4^{\circ} \mathrm{C}$ for $2 \mathrm{~h}$ and centrifuged at $4^{\circ} \mathrm{C}$ and $3,000 \mathrm{~g}$ for $10 \mathrm{~min}$, and $100 \mu \mathrm{L} /$ tube of the serum was stored at $-80^{\circ} \mathrm{C}$.

Metabolite extraction for LC-MS detection. The samples were thawed at $4^{\circ} \mathrm{C}$ on ice. Then, $100 \mu \mathrm{L}$ of each sample was obtained and placed in an EP tube, extracted with $400 \mu \mathrm{L}$ of extraction solvent (1:1 V methanol:V acetonitrile), vortexed for $30 \mathrm{~s}$, ultrasound treated for $10 \mathrm{~min}$ (while being cooled in ice water), and next chilled for $1 \mathrm{~h}$ at $-20^{\circ} \mathrm{C}$ to precipitate the proteins. The samples were then centrifuged at $12,000 \mathrm{~g}$ for $15 \mathrm{~min}$ at $4^{\circ} \mathrm{C}$. The supernatant $(425 \mu \mathrm{L})$ was transferred into fresh EP tubes, the extracts were dried in a vacuum concentrator without heating, and $100 \mu \mathrm{L}$ of extraction solvent was added to reconstitute the samples. The samples were vortexed for $30 \mathrm{~s}$, sonicated for $10 \mathrm{~min}$ (in a $4^{\circ} \mathrm{C}$ water bath), and centrifuged again at $12,000 \mathrm{~g}$ for $15 \mathrm{~min}$ at $4^{\circ} \mathrm{C}$. A $60 \mu \mathrm{L}$ volume of supernatant was transferred into fresh $2 \mathrm{~mL}$ LC/MS glass vials, then $10 \mu \mathrm{L}$ was taken from each sample and pooled as QC samples. Finally, $60 \mu \mathrm{L}$ of the supernatant was obtained for the UHPLC-QTOF-MS analysis.

LC-MS/MS detection and data processing. LCMS/MS analyses were performed using a 1290 Infinity II UHPLC system (Agilent Technologies, Germany) with an Acquity UPLC BEH Amide column $(1.7 \mu \mathrm{m}$, $2.1 \times 100 \mathrm{~mm}$; Waters, USA) coupled to a Q-TOF TripleTOF 6600 time-of-flight mass analyser (AB Sciex, Singapore). The mobile phase consisted of $25 \mathrm{mM}$ of $\mathrm{NH}_{4} \mathrm{Ac}$ and $25 \mathrm{mM}$ of $\mathrm{NH}_{4} \mathrm{OH}$ in water $(\mathrm{pH} 9.75)$ as $\mathrm{A}$ and acetonitrile as $\mathrm{B}$ and it was carried out with an elution gradient as follows: $0 \mathrm{~min}-95 \% \mathrm{~B}, 0.5 \mathrm{~min}$ $-95 \%$ B, 7 min $-65 \%$ B, 8 min $-40 \%$ B, 9 min $-40 \%$ B, $9.1 \mathrm{~min}-95 \% \mathrm{~B}$, and $12 \mathrm{~min}-95 \% \mathrm{~B}$. The delivery rate was $0.5 \mathrm{~mL} / \mathrm{min}$ and the injection volume was $1 \mu \mathrm{L}$. In each cycle, 12 precursor ions with intensity greater than 100 were selected for fragmentation at a collision energy of $30 \mathrm{~V}$ (15 MS/MS events with a product ion accumulation time of $50 \mathrm{~ms}$ each). The electrospray ionisation source conditions were set as follows: ion source gas 160 PSI pressure, ion source gas 260 PSI, curtain gas $35 \mathrm{PSI}$, source temperature $650^{\circ} \mathrm{C}$, and ion spray voltage floating (ISVF) of $5,000 \mathrm{~V}$ in positive or $-4,000 \mathrm{~V}$ in negative mode.

The MS raw data (wiff) files were converted to the mzXML format using ProteoWizard (1) and processed with R package XCMS (version 3.2) (15). The preprocessing results formed a data matrix that consisted of the retention time $(\mathrm{RT})$, mass-to-charge ratio $(\mathrm{m} / \mathrm{z})$ values, and peak intensity. The $\mathrm{R}$ package CAMERA was used for peak annotation after XCMS data processing. An in-house MS2 database was the source of metabolite identification.

The ionisation source of the LC-QTOFMS platform is electrospray ionisation, and there are two ionisation modes: positive and negative. In this experiment, both ionisation modes were used together for metabolomic detection.

MRM target metabolomics detection. UHPLC separation was carried out using the Agilent 1290 Infinity II series UHPLC System equipped with the Acquity UPLC BEH Amide column. The mobile phase A was $10 \mathrm{mmol} / \mathrm{L}$ ammonium formate $/ 0.1 \%$ formic acid (the mobile phase A of L-glutamic acid was $1 \%$ formic acid in water) and mobile phase B was acetonitrile (the mobile phase B of L-glutamic acid was $1 \%$ formic acid in acetonitrile). The column temperature was set at $35^{\circ} \mathrm{C}$, the auto-sampler temperature at $4{ }^{\circ} \mathrm{C}$, and the injection volume was $1 \mu \mathrm{L}$. A 6460 triple quadrupole mass spectrometer (Agilent Technologies, USA), equipped with an Agilent Jet Stream electrospray ionisation interface was applied for assay development. The ion source parameters were capillary voltage of $+4,000 /-3,500 \mathrm{~V}$, nozzle voltage of $+500 /-500 \mathrm{~V}$, gas $\left(\mathrm{N}_{2}\right)$ temperature of $300^{\circ} \mathrm{C}$, gas $\left(\mathrm{N}_{2}\right)$ flow of $5 \mathrm{~L} / \mathrm{min}$, sheath gas $\left(\mathrm{N}_{2}\right)$ temperature of $250^{\circ} \mathrm{C}$ and flow of $11 \mathrm{~L} / \mathrm{min}$, and nebulizer pressure of $45 \mathrm{psi}$. Agilent MassHunter BioConfirm workstation software version B.08.00 (Agilent Technologies, USA) was employed for MRM data acquisition and processing.

Data analysis. A multivariate statistical analysis with an unsupervised principal component analysis (PCA) was used to reveal the internal structure and overall distribution of the data. Orthogonal projections to latent structures discriminant analysis (OPLS-DA) was used to filter out the metabolites that were not related to the classification variables. Student's $t$-test was used for the univariate statistical analysis. In this study, differential metabolites were screened by combining the P value obtained from the Student's $t$-test and variable importance in projection (VIP) of the first principal component of the OPLS-DA model, and the screened differential metabolites were subsequently analysed by hierarchical clustering analysis.

Metabolic pathway analysis. The Kyoto Encyclopaedia of Genes and Genomes (KEGG) database was used to search for metabolic pathways related to different metabolites. Metaboanalyst 4.0 (http://www.metaboanalyst.ca/) was used to analyse those pathways. 
Table 1. Experimental and control group animal basic information

\begin{tabular}{cccccc}
\hline No. & Variety & $\begin{array}{c}\text { Age } \\
\text { (years) }\end{array}$ & Gender & Spaying status & Tumour type \\
\hline T-1 & Chinese pastoral cat & 10 & Female & Unspayed & Mammary gland carcinoma \\
T-2 & Chinese pastoral cat & 10 & Female & Unspayed & Mammary gland carcinoma \\
T-3 & Chinese pastoral cat & 9 & Female & Unspayed & Mammary gland carcinoma \\
T-4 & Chinese pastoral cat & 9 & Female & Unspayed & Mammary gland carcinoma \\
T-5 & Chinese pastoral cat & 8 & Female & Unspayed & Mammary gland carcinoma \\
T-6 & Chinese pastoral cat & 7 & Female & Unspayed & Mammary gland carcinoma \\
C-1 & Chinese pastoral cat & 10 & Female & Unspayed & Healthy control \\
C-2 & Chinese pastoral cat & 9 & Female & Unspayed & Healthy control \\
C-3 & Chinese pastoral cat & 9 & Female & Unspayed & Healthy control \\
C-4 & Chinese pastoral cat & 8 & Female & Unspayed & Healthy control \\
C-5 & Chinese pastoral cat & 8 & Female & Unspayed & Healthy control \\
C-6 & Chinese pastoral cat & 8 & Female & Unspayed & Healthy control \\
\hline
\end{tabular}

\section{Results}

Multivariate statistical analysis results. PCA analysis was performed on two groups of samples. As seen in Fig. 1A and Fig. 1B, both groups of samples are in the $95 \%$ confidence interval with significant separation between groups.

The OPLS-DA score is shown in Fig. 2A and Fig. 2B. The results revealed a highly significant difference between the two groups of samples, and all samples were within the $95 \%$ confidence interval.

Screening of differential metabolites. The card value standards used in this test were $\mathrm{P}<0.05$ and VIP $>1$. The screening results are presented in Fig. 3A and Fig. 3B. Each point in the figures represents a metabolite. The size of the scatter increases in proportion to the VIP value and its colour represents the final screening results. Metabolites that are significantly up regulated are shown in red, metabolites that are significantly down regulated are shown in blue, and metabolites that are not significantly different are shown in grey.

Using qualitative analysis of the selected differential metabolites, 96 were identified in the NEG mode. Compared with group $\mathrm{C}$, there were 24 down regulated and 72 up regulated metabolites in group $\mathrm{T}$. In the POS mode, 103 metabolites were identified. Compared with group $\mathrm{C}$, there were 20 down regulated and 83 up regulated metabolites in group T. It was found that the smaller the P value and the larger the VIP value, the more significant the difference was in the substance amount. Some metabolites with significant differences are listed, and their specific changes are shown in Table 2. The qualitative differential metabolites underwent hierarchical clustering analysis (HCA), and the results are presented in Fig. 4A and Fig. 4B. The results of the HCA analysis revealed that the metabolites were significantly differentiated between the two groups.

Differential metabolite pathway analysis. The differential metabolites were mapped to the KEGG database. Based on a further comprehensive analysis of all metabolic pathways (including enrichment and topological analyses), the key pathways with the highest correlation with feline mammary carcinomas were selected. In the NEG mode, differential metabolites were primarily involved in biological metabolic processes, including metabolisms of purine, glycerolipid, alanine, aspartate, glutamate, nitrogen, phenylalanine, $\beta$-alanine, and histidine, the citrate cycle (the tricarboxylid acid (TCA) cycle), pantothenate and coenzyme A biosynthesis, vitamin B6 metabolism, and other biological processes. In the POS mode, the differential metabolites were primarily involved in biological metabolic processes including glycine, serine, and threonine metabolisms, fatty acid biosynthesis, metabolisms of arginine, proline, pyrimidine, purine, glycerophospholipid, glutathione, linoleic acid, alanine, aspartate and glutamate, and arachidonic acid, and other biological processes.

The results of the metabolic pathway analysis are presented in a bubble diagram (Fig. 5A and Fig. 5B). Each bubble represents a metabolic pathway. The abscissa and size of the bubble indicate the degree of influence of the path in the topology analysis. It was observed that the larger the bubble, the greater the influence factor. The ordinate and colour of the bubble represent the $\mathrm{P}$ value in the enrichment analysis (the negative natural logarithm (i.e. $-\ln \mathrm{P}$ value). The darker the colour, the smaller the $\mathrm{P}$ value, and the more significant the degree of enrichment.

The metabolic pathways involved in differential metabolites were analysed according to the degree of influence and enrichment of metabolic pathways and the role of different metabolites in the development of feline mammary carcinomas. Six marker differential metabolites closely related to the molecular mechanism of feline mammary carcinomas were screened. Among them, inosine, succinate, L-alanine, and L-glutamate were down regulated, while hypoxanthine and adenine were up regulated. The interaction network between the six marker differential metabolites is schematised in Fig. 6.

MRM target metabolomics validation. The MRM target metabolomics verification results are presented in Table 3. The results of LC-MS metabolomics are consistent with those of the MRM target metabolomics, which indicates that the LC-MS metabolomic results are accurate and reliable. 
A

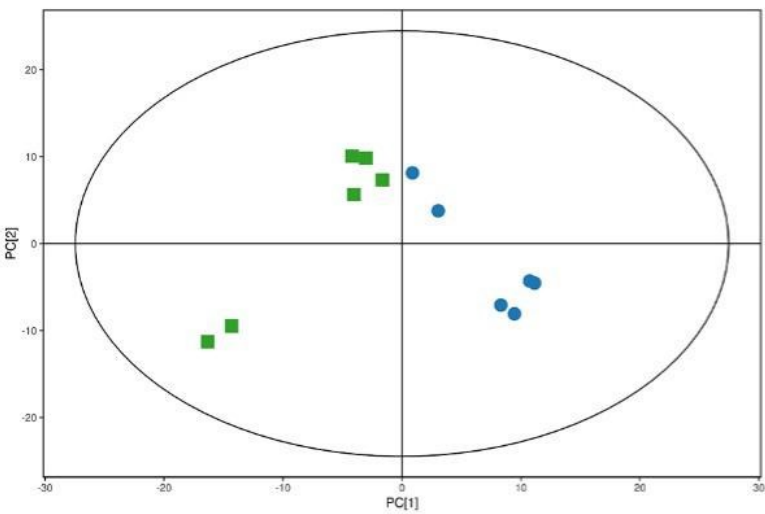

B

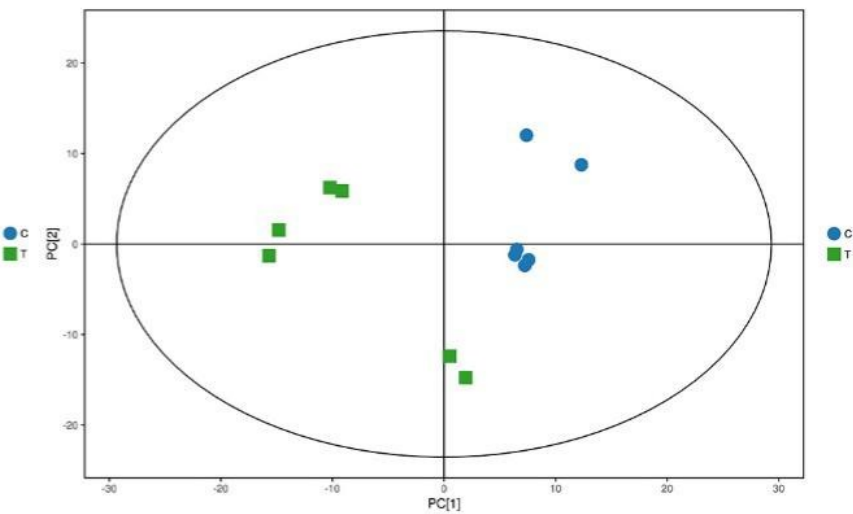

Fig. 1. PCA score diagram in NEG mode and POS mode

In the figure, the ellipse represents the $95 \%$ confidence interval, and the abscissa PC [1] and ordinate PC [2] represent the score of the principal component ranking first and second, respectively. The scatter shapes and colours represent the different experimental groups

A

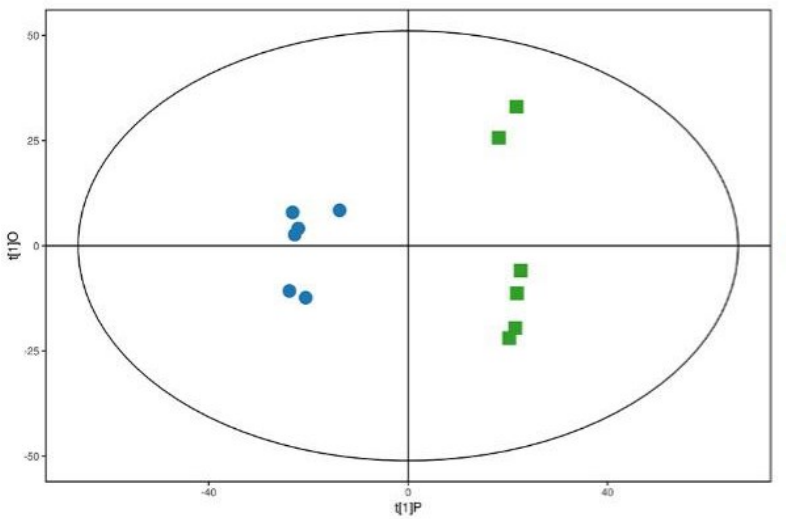

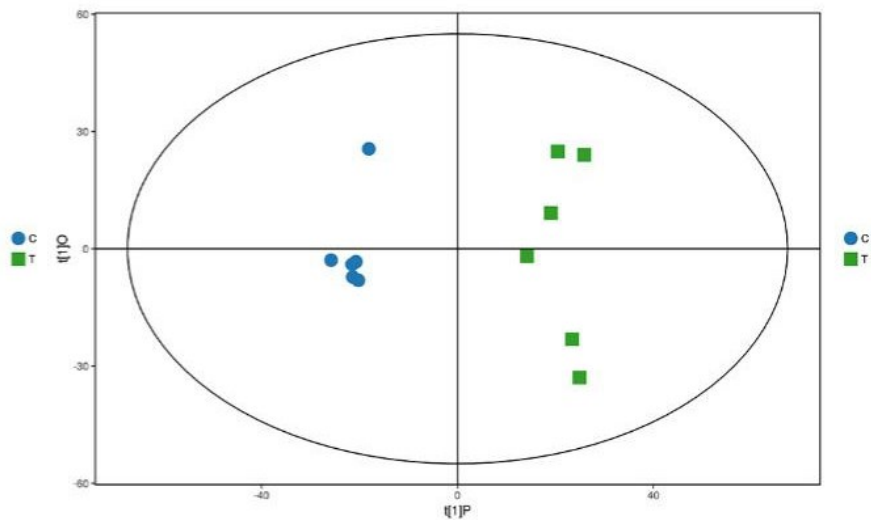

Fig. 2. OPLS-DA score in the NEG mode and POS mode

In the figure, the ellipse represents the $95 \%$ confidence interval, and the abscissa t[1]P represents the predicted principal component score of the first principal component. The ordinate $t[1] \mathrm{O}$ represents the orthogonal principal component score, and the scatter shapes and colours represent the different experimental groups

A

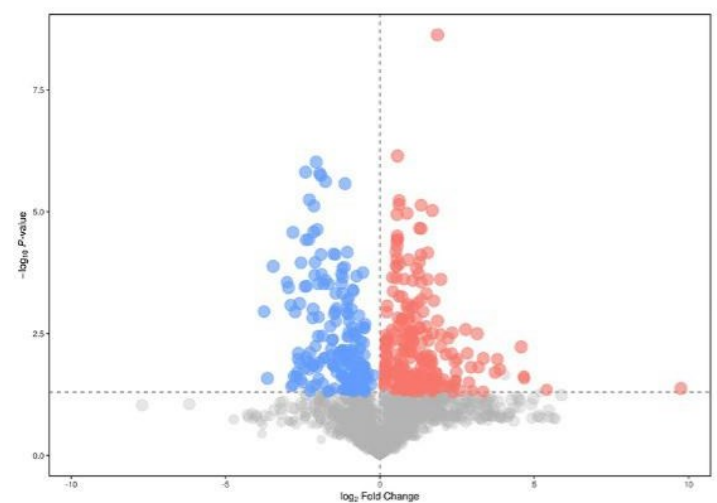

B

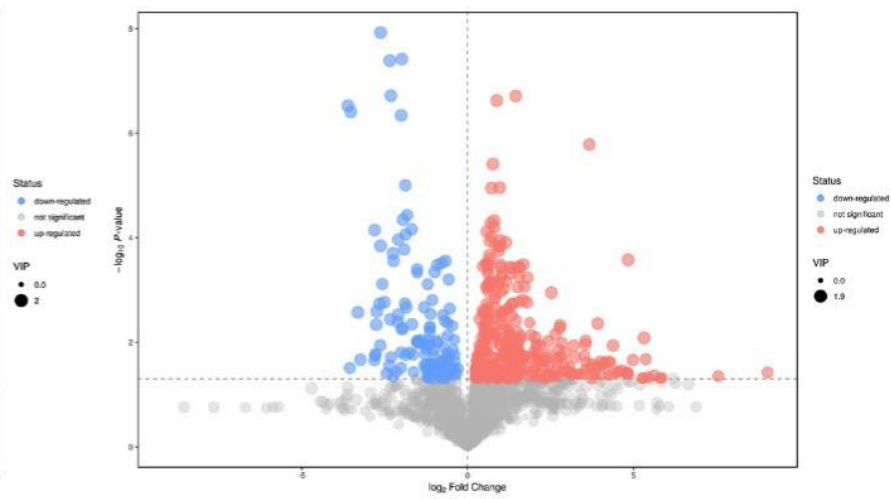

Fig. 3. Volcano map of NEG (A) and POS (B) modes differential metabolite screening

The abscissa represents the fold-change of the group comparing each substance (taking the logarithm base 2). The ordinate represents the P-value of the Student's $t$-test (taking the negative logarithm base 10) 
Table 2. Differential metabolites in feline mammary carcinomas based on LC-MS

\begin{tabular}{|c|c|c|c|c|c|}
\hline No. & Substance name & VIP & $\mathrm{P}$ & $\begin{array}{l}\text { Variation } \\
\text { trend }\end{array}$ & Detection mode \\
\hline 1 & L-glutamate & 1.906599493 & $9.39957 \mathrm{E}-06$ & $\uparrow$ & NEG \\
\hline 2 & L-alanine & 1.870339069 & $9.66197 \mathrm{E}-05$ & $\uparrow$ & NEG \\
\hline 3 & Glycerol 3-phosphate & 1.813604415 & 0.001128205 & $\uparrow$ & NEG \\
\hline 4 & Succinate & 1.790389164 & 0.000554729 & $\uparrow$ & NEG \\
\hline 5 & 20-hydroxy-PGE2 & 1.786772601 & $2.17846 \mathrm{E}-05$ & $\uparrow$ & NEG \\
\hline 6 & Fosfomycin & 1.779219395 & 0.002655475 & $\uparrow$ & NEG \\
\hline 7 & 3-methyluridine & 1.764311315 & 0.000277439 & $\uparrow$ & NEG \\
\hline 8 & $\mathrm{~N}$-acetyl-L-alanine & 1.720196947 & 0.000240845 & $\uparrow$ & NEG \\
\hline 9 & Choline & 1.877285980 & $2.37754 \mathrm{E}-07$ & $\uparrow$ & POS \\
\hline 10 & Trigonelline & 1.822938835 & 0.001548866 & $\uparrow$ & POS \\
\hline 11 & Ile-asn & 1.806880095 & $1.10469 \mathrm{E}-05$ & $\uparrow$ & POS \\
\hline 12 & Arachidonic acid (peroxide free) & 1.772611472 & 0.001126785 & $\uparrow$ & POS \\
\hline 13 & S-methyl-5'-thioadenosine & 1.770146402 & 0.000360333 & $\uparrow$ & POS \\
\hline 14 & Creatinine & 1.751825211 & $5.23436 \mathrm{E}-05$ & $\uparrow$ & POS \\
\hline 15 & L-histidinol & 1.74002669 & 0.000841015 & $\uparrow$ & POS \\
\hline 16 & Guanidine acetic acid & 1.698257167 & 0.000546455 & $\uparrow$ & POS \\
\hline 17 & Cytosine & 1.662976359 & 0.000112223 & $\uparrow$ & POS \\
\hline 18 & Inosine & 1.481481111 & 0.002446273 & $\downarrow$ & NEG \\
\hline 19 & Adenine & 1.792517828 & 0.001575227 & $\uparrow$ & NEG \\
\hline 20 & Hypoxanthine & 1.730804616 & 0.00067303 & $\uparrow$ & POS \\
\hline
\end{tabular}

Table 3. MRM target metabolomic validation

\begin{tabular}{lcccccc}
\hline \multicolumn{1}{c}{ Substance name } & \multicolumn{2}{c}{ LC-MS non-target metabolomics results } & & MRM target metabolomics results \\
\hline & Fold change & VIP & P & $\begin{array}{c}\text { Variation } \\
\text { trend } \\
\text { (T C) }\end{array}$ & $\begin{array}{c}\text { T group final } \\
\text { concentration } \\
\text { (average) }\end{array}$ & $\begin{array}{c}\text { C group final } \\
\text { concentration } \\
\text { (average) }\end{array}$ \\
\hline S-methyl-5'-thioadesine & 2.879152807 & 1.770146402 & 0.000360333 & $\uparrow$ & 0.011 & 0.003 \\
Creatinine & 1.632513451 & 1.751825211 & $5.23436 \mathrm{E}-05$ & $\uparrow$ & 42.117 & 1.383 \\
Cytosine & 1.981265029 & 1.662976359 & 0.000112223 & $\uparrow$ & 4.381 & 0.49 \\
Choline & 1.836734369 & 1.877285980 & $2.37754 \mathrm{E}-07$ & $\uparrow$ & 1.915 & 0.71 \\
Betaine nicotinate & 3.178675095 & 1.822938835 & 0.001548866 & $\uparrow$ & 190.229 \\
(Trigonelline) & 1.891848591 & 1.870339069 & $9.66197 \mathrm{E}-05$ & $\uparrow$ & 30.959 \\
L-alanine & 5.129593730 & 1.533880516 & 0.019410600 & $\uparrow$ & 94.83 \\
L-glutamic acid & & & & & \\
\hline
\end{tabular}

Fold change $>1$ indicates that the substance is up regulated in group T compared with group $\mathrm{C}$. Fold change $<1$ means that the substance is down regulated in group $\mathrm{T}$ compared with group $\mathrm{C}$

A

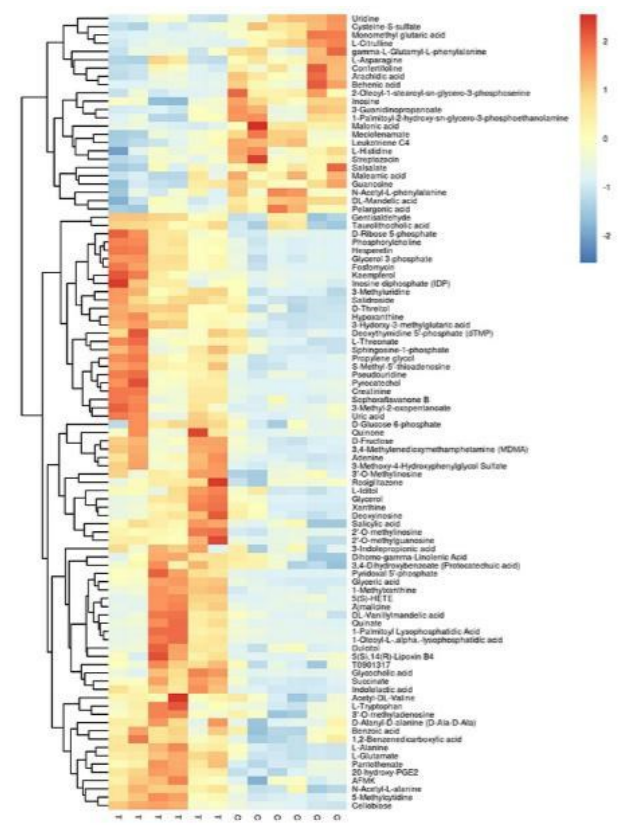

B

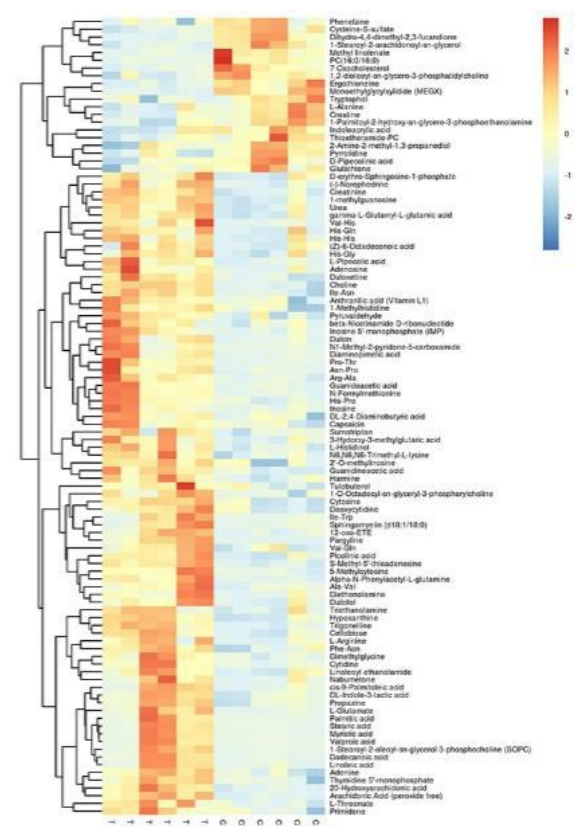

Fig. 4. HCA analysis results in NEG (A) and POS (B) modes 
A

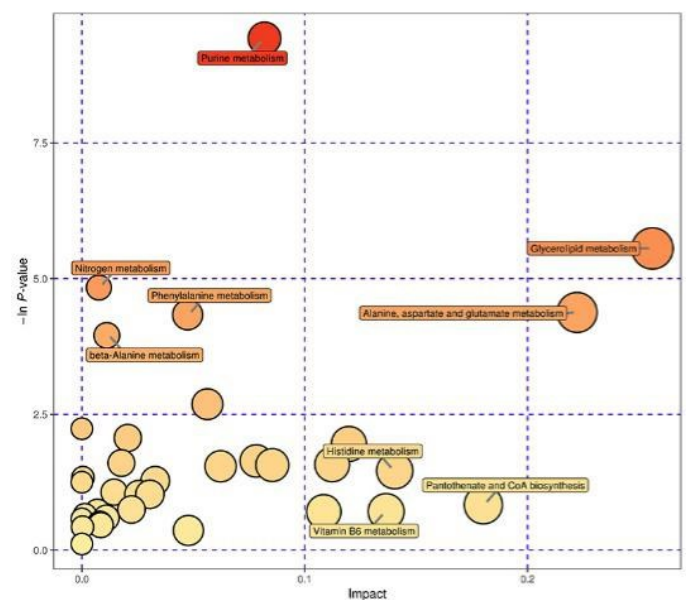

B

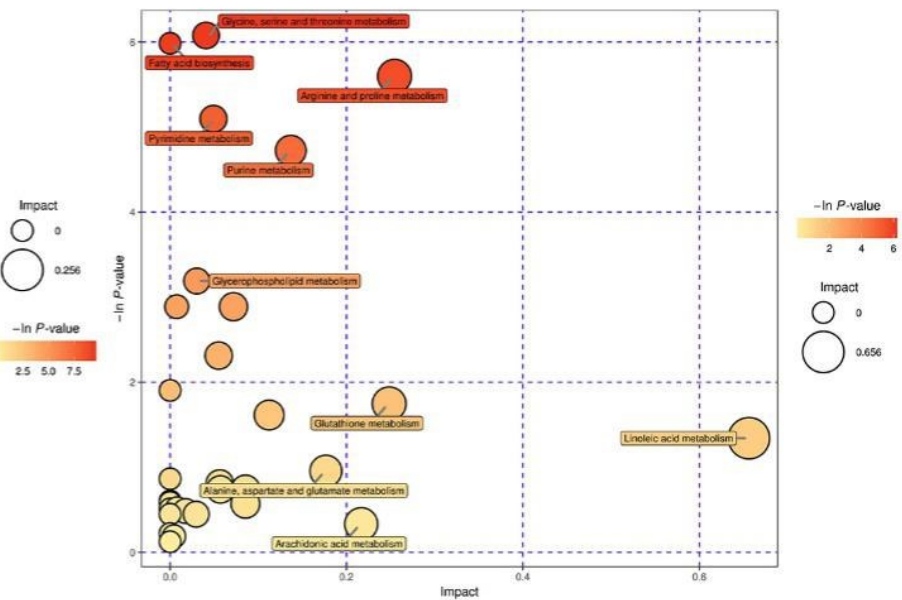

Fig. 5. Bubble diagram of the metabolic pathway analysis in NEG and POS mode

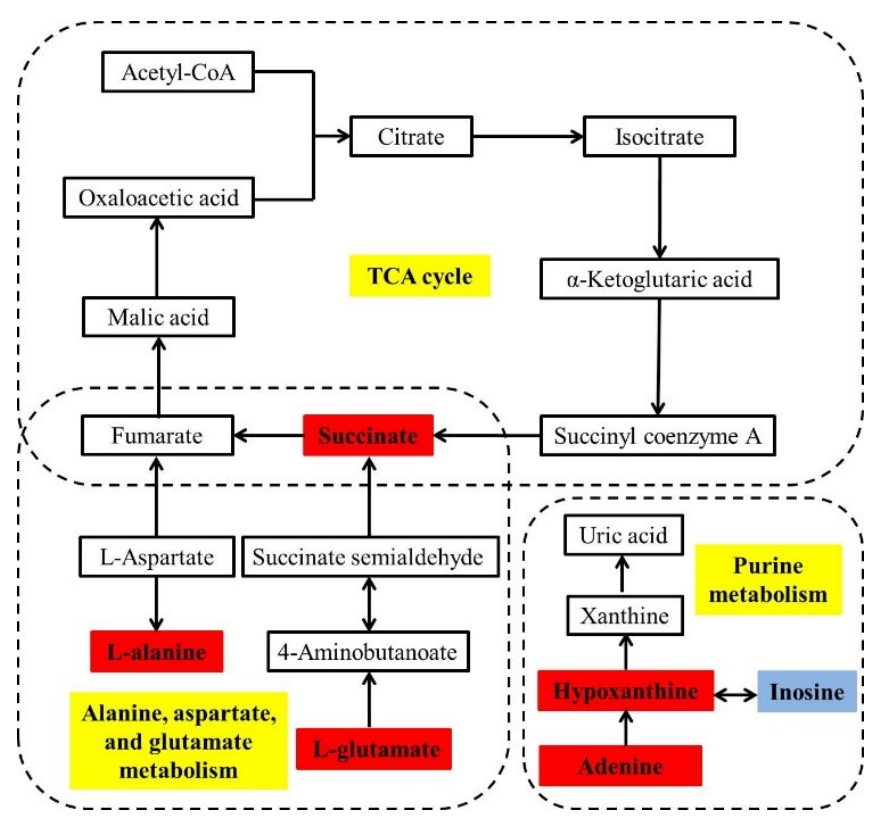

Fig. 6. Interaction network diagram of six biomarker differential metabolites. Red indicates that the metabolites are up regulated in group $\mathrm{T}$, blue indicates that the metabolite is down regulated in group $\mathrm{T}$, yellow indicates metabolic pathways, and metabolites without a coloured background are the upstream and downstream substances that are differentially metabolised

\section{Discussion}

In this study, the key differential metabolic pathways included alanine, aspartate, and glutamate metabolisms, the TCA cycle, and purine metabolism. A large amount of research data has shown that a capacity for infinite proliferation is a characteristic of cancer cells. Therefore, when cancer occurs in the body, the levels of substances related to cell proliferation and metabolism will change dramatically. Amino acids provide the basis of enzymes, receptors, antibodies, signalling molecules, hormones, and several other basic protein structures in all organisms. The TCA cycle is a key metabolic pathway through which mammalian cells utilise glucose, amino acids, and fatty acids. Purine is a basic component of nucleotides during cell proliferation, and its metabolism is associated with biochemical reactions, including the metabolism of cell cycle immune functionality and signal transduction. Therefore, these three metabolic pathways are closely related to the occurrence of cancer. In this study, according to the $\mathrm{P}$ value, the VIP value, and the key pathways of the substance, six landmark differential metabolites found to be closely related to the occurrence of cat mammary cancer were selected.

Abnormal amino acid metabolism. The results showed that the expression of L-glutamate and L-alanine in group $\mathrm{T}$ was significantly up regulated. L-glutamate 
and L-alanine are both important sugar-producing amino acids which are primarily involved in the alanine, aspartate, and glutamate metabolism pathways. When cancer cells proliferate indefinitely, large amounts of energy-associated substances are required, leading to an increase in sugar production. This may be the main reason for the observed increase in the L-glutamate and L-alanine content in group T. Alanine is converted from pyruvate, which is the intermediate substance produced by glucose fermentation. Pyruvate can be converted into acetyl coenzyme $\mathrm{A}$, and transformation among the three major sugar, fat, and protein substances in the body can be achieved through the TCA cycle (16). In addition, during the metabolism of breast cancer, ammonia produced by the body will be recycled into the metabolism of amino acids. The expression of glutamate dehydrogenase (GDH) and glutamine synthetase (GS) will also increase in breast cancer, leading to increased glutamate content in the body (18). In tumour development, the organism's DNA, protein, and lipid synthesis processes are significantly increased. L-glutamate and L-alanine are the basic units of protein synthesis, which are required for the abnormal gene translation and expression in the tumour. This may also be one of the reasons for the increased glutamate and alanine expression in the $\mathrm{T}$ group. There are also studies that have shown that the number and type of amino acids may affect the muscle metabolism of cachexia patients, and that the maintenance of the amino acid content at a certain level can delay the decline of muscle metabolism in these patients (14). Therefore, the upregulation of the amino acid content in breast cancer may also be a manifestation of a mechanism to counter muscle reduction and maintain muscle function.

Abnormal glycometabolism. The results showed that the level of succinate expression was up regulated in group $\mathrm{T}$ and involved in the TCA cycle metabolic pathway. The TCA cycle regulates energy generation in mitochondrial respiration and plays a key role in carbohydrate metabolism. Moreover, the TCA cycle is the most effective way for organisms to obtain energy. Although the TCA cycle can provide $90 \%$ of the energy required for normal cells, it can only produce $50 \%$ of the required energy in cancer cells (20). Succinyl coenzyme A produces succinate when catalysed by a TCA cycle enzyme, and succinate plays an important role in mitochondrial energy metabolism. However, abnormal mitochondrial metabolism may lead to the accumulation of extracellular and/or cytoplasmic succinate (19). This may be the reason for the observed increase in the succinate content in group T. In addition, some studies have shown that succinate is a novel type of cancerpromoting factor secreted by cancer cells (21). Moreover, succinate can trigger macrophages to polarise into tumour-associated macrophages, as well as promote tumour metastasis and invasion. This may also be a reason for the increased succinate content in group $\mathrm{T}$. Also, some studies have shown that the urine content of succinate from patients with ovarian cancer is higher than that of normal people, and succinate is defined as a potential marker of ovarian cancer $(9,23)$. In metabolomics tests of gastric cancer and colon cancer patients, succinate was also increased (8).

Abnormal purine metabolism. The results showed that inosine expression was down regulated. In addition, adenine and hypoxanthine were up regulated in the $\mathrm{T}$ group and were involved in the purine metabolism pathway. Inosine is a component of coenzyme A, adenosine triphosphate, deoxyribonucleic acid, and ribonucleic acid in the body, and also participates in the metabolism of various substances and energy. In the process of breast cancer cell proliferation, an abundance of energy is consumed and with it so is inosine for the production of multiple nucleotides. In the $\mathrm{T}$ group, the inosine content was found to be down regulated, which may be due to increased energy metabolism and the rapid increase of nucleotide production, resulting in excessive inosine consumption and down regulation. In a study of the serum and plasma metabolomics in lung cancer, inosine was down regulated and was proposed as a serum biomarker of lung cancer (10). Adenine participates in a variety of cellular processes. When cancer occurs, cell proliferation accelerates, and DNA synthesis will increase. At this time, a large amount of adenine will be produced in the body. Therefore, the up regulation of the adenine content observed in this study may be the result of accelerated cell proliferation. Adenine has been explored for pharmacological use. Some studies have shown that adenine can inhibit the growth of colon cancer cells and attributed anticancer effects to this natural compound (11). Hypoxanthine can be formed by different deamination mechanisms during conditions of chronic inflammation, and it can be produced when adenine deamination causes DNA damage. In this study, the occurrence of feline mammary carcinomas was accompanied by chronic inflammatory processes and increased adenine content, which may be the cause of the increased hypoxanthine content. Hypoxanthine is also a potential cause of colon, liver, pancreatic, bladder, and gastric diseases (5).

Six metabolites that were closely related to feline mammary carcinomas were screened: L-glutamate, L-alanine, succinate, adenine, hypoxanthine, and inosine. The expression of all but the last metabolite was upregulated and expression of inosine alone was down regulated. At the same time, alanine, aspartate, and glutamate metabolism, the TCA cycle, and purine metabolism, representing three important metabolic pathways, were also interpreted. These differential metabolites are involved in multiple amino acid metabolism, the TCA cycle, and purine metabolism processes. This result suggests that the body will mobilize a large number of substances to participate in processes such as energy metabolism and cell biosynthesis during the occurrence of FMC. These findings provide a new direction for further study of the mechanisms associated with cat mammary neoplasms in the future. 
* These authors contributed equally to the work and should be considered co-first authors.

Conflict of Interests Statement: The authors declare that there is no conflict of interests regarding the publication of this article.

Financial Disclosure Statement: This study was supported by grants from the Heilongjiang Province Postdoctoral Science Foundation (grant LBH-Z18257), Heilongjiang Bayi Agricultural University Postdoctoral Science Foundation, the National Key Research and Development Program of China (grant 2016YFD0501008), Heilongjiang Bayi Agricultural University Postgraduate Research Innovation Funding Program (grant YJSCX2018-Y23), and the Heilongjiang Bayi Agricultural University Support Program for San Heng San Zong (grant TDJH201903).

Animal Rights Statement: The animal experiments were approved by the Institutional Animal Care and Use Committee (IACUC). All experimental procedures were performed in accordance with the regulations of the Administration of Affairs Concerning Experimental Animals approved by the School Council of Heilongjiang Bayi Agricultural University of China Daqing. The study protocol was approved by the Ethics Committee on the Use and Care of Animals of Heilongjiang Bayi Agricultural University (Daqing, China).

\section{References}

1. Chambers M.C., MacLean B., Burke R., Amode D., Ruderman D.L., Neumann S., Gatto L., Fischer B., Pratt B., Egertson J., Hoff K., Kessner D., Tasman N., Shulman N., Frewen B., Baker T.A., Brusniak M.-Y., Paulse C., Creasy D., Flashner L., Kani K., Moulding C., Seymour S.L., Nuwaysir L.M., Lefebvre B., Kuhlmann F., Roark J., Rainer P., Detlev S., Hemenway T., Huhmer A., Langridge J., Connolly B., Chadick T., Holly K., Eckels J., Deutsch E.W., Moritz R.L., Katz J.E., Agus D.B., MacCoss M., Tabb D.L., Mallick P.: A cross-platform toolkit for mass spectrometry and proteomics. Nature Biotechnology 2012, 30, 918-920.

2. Chang Y.C., Chuang H. L., Yin J.H., Liao J.W., Chen T.H., Wang Y.C.: Significance of sphingosine kinase 1 expression in feline mammary tumors. BMC Vet Res 2019, 15, 155

3. Cunha S., Corgozinho K., Justen H., Silva K., Leite J., Ferreira A.M.: Survival and disease-free interval of cats with mammary carcinoma treated with chain mastectomy. Acta Sci Vet 2016, 44, $1-8$.

4. Dagher E., Abadie J., Loussouarn D., Campone M., Nguyen F.: Feline invasive mammary carcinomas: prognostic value of histological grading. Vet Pathol 2019, 56, 660-670.

5. DeVito S., Woodrick J., Song L., Roy R.: Mutagenic potential of hypoxanthine in live human cells. Mutat Res Fund Mol Mech Mutagen 2017, 803, 9-16.

6. Ferreira D., Martins B., Soares M., Correia J., Adega F., Ferreira F., Chaves R.: Gene expression association study in feline mammary carcinomas. PLoS One 2019, 14, e0221776.
7. Gundim L.F., Wilson T.M., Soares N.P., Castro I.P., Castro M.R., Medeiros-Ronchi A.A.: Her-2 expression in feline mammary lesions. Acta Vet Brno 2019, 88, 43-47.

8. Hirayama A., Kami K., Sugimoto M., Sugawara M., Toki N., Onozuka H., Kinoshita T., Saito N., Ochiai A., Tomita M., Esumi H., Soga T.: Quantitative metabolome profiling of colon and stomach cancer microenvironment by capillary electrophoresis time-of-flight mass spectrometry. Cancer Res 2009, 69, 4918-4925.

9. Jiang T., Lin Y.L., Yin H.Q., Wang S.S., Sun Q.L., Zhang P.H., Bi W.X.: Correlation analysis of urine metabolites and clinical staging in patients with ovarian cancer. Int J Clin Exp Med 2015, $8,18165-18171$

10. Kumar N., Shahjaman M., Mollah M.N., Islam S.M.S., Hoque M.A.: Serum and plasma metabolomic biomarkers for lung cancer. Bioinformation 2017, 13, 202-208

11. Lai H.W., Wei J.C.C., Hung H.C., Lin C.C.: Adenine inhibits the growth of colon cancer cells via AMP-activated protein kinase mediated autophagy. Evid Based Complement Alternat Med 2019, 2019, 9151070.

12. Maniscalco L., Guil-Luna S., Iussich S., Gattino F., Trupia C., Millan Y., Martín de las Mulas J., Sánchez-Céspedez R., Saeki K. Accornero P., De Maria F.: Expression of the short form of RON/STK in feline mammary carcinoma. Vet Pathol 2019, 56, 220-229.

13. Mills S.W., Musil K.M., Davies J.L., Hendrick S., Duncan C., Jackson M.L., Kidney B., Philibert H., Wobeser B.K., Simko E.: Prognostic value of histologic grading for feline mammary carcinoma: a retrospective survival analysis. Vet Pathol 2015, 52, 238-249.

14. Pötgens S.A., Sboarina M., Bindels L.B.: Polyunsaturated fatty acids, polyphenols, amino acids, prebiotics: can they help to tackle cancer cachexia and related inflammation? Curr Opin Clin Nutr Metab Care 2018, 21, 458-464.

15. R Core Team: R: A language and environment for statistical computing. R Foundation for Statistical Computing, Vienna, 2017. https://www.R-project.org/.

16. Smilowitz J.T., O’Sullivan A., Barile D., German B., Lönnerdal B., Slupsky C.M.: The human milk metabolome reveals diverse oligosaccharide profiles. J Nutr 2013, 143, 1709-1718.

17. Soares M., Madeira S., Correia J., Peleteiro M., Cardoso F., Ferreira F.: Molecular based subtyping of feline mammary carcinomas and clinicopathological characterization. Breast 2016, $27,44-51$.

18. Spinelli J.B., Yoon H., Ringel A.E., Jeanfavre S., Clish C.B. Haigis M.C.: Metabolic recycling of ammonia via glutamate dehydrogenase supports breast cancer biomass. Science 2017 , 358, 941-946.

19. Tretter L., Patocs A., Chinopoulos C.: Succinate, an intermediate in metabolism, signal transduction, ROS, hypoxia, and tumorigenesis. BBA - Bioenergetics 2016, 1857, 1086-1101.

20. Vasseur S., Tomasini R., Tournaire R., Iovanna J.L.: Hypoxia induced tumor metabolic switch contributes to pancreatic cancer aggressiveness. Cancers 2010, 2, 2138-2152.

21. Wu J.Y., Huang T.W., Hsieh Y.T., Wang Y.F., Yen C.C., Lee G.L. Cancer-derived succinate promotes macrophage polarization and cancer metastasis via succinate receptor. Molecular Cell 2020, 77 213-227.

22. Zappulli V., Rasotto R., Caliari D., Mainenti M., Peña L., Goldschmidt M.H., Kiupel M.: Prognostic evaluation of feline mammary carcinomas: a review of the literature. Vet Pathol 2015, $52,46-60$.

23. Zhang T., Wu X.Y., Ke C.F., Yin M.Z., Li Z.Z., Fan L.J., Zhang W., Zhang H.Y., Zhao F.L., Zhou X.H., Lou G., Li K.: Identification of potential biomarkers for ovarian cancer by urinary metabolomic profiling. J Proteome Res 2013, 12, $505-512$. 